\title{
Barkhausen pulses caused by domain merging in congruent lithium niobate
}

Cite as: Appl. Phys. Lett. 117, 022903 (2020); https://doi.org/10.1063/5.0014220

Submitted: 18 May 2020 . Accepted: 04 July 2020. Published Online: 15 July 2020

A. R. Akhmatkhanov (D), I. A. Kipenko, A. A. Esin (D), and V. Ya. Shur (D)

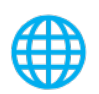

\section{ARTICLES YOU MAY BE INTERESTED IN}

Chemical renormalization of the paraelectric-ferroelectric phase transition in $\mathrm{PbTiO}_{3}-\mathrm{BiB}_{0.5}^{\prime} B_{0.5}^{\prime \prime} \mathrm{O}_{3}$ solid solutions with tetragonal symmetry

Applied Physics Letters 117, 022904 (2020); https://doi.org/10.1063/5.0013491

Avalanches from charged domain wall motion in $\mathrm{BaTiO}_{3}$ during ferroelectric switching APL Materials 8, 011105 (2020); https://doi.org/10.1063/1.5128892

Study of ferroelectric characteristics of $\mathrm{Hf}_{0.5} \mathrm{Zr}_{0.5} \mathrm{O}_{2}$ thin films grown on sputtered or atomiclayer-deposited TiN bottom electrodes

Applied Physics Letters 117, 022902 (2020); https://doi.org/10.1063/5.0011663
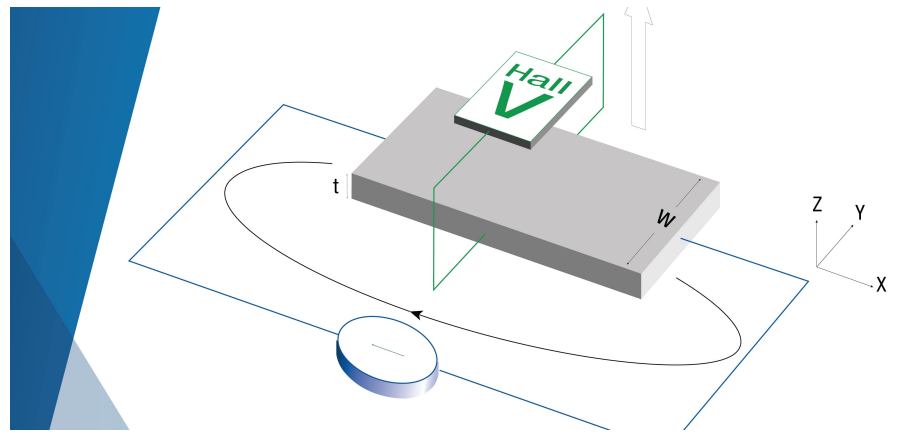

Tips for minimizing Hall measurement errors Download the Technical Note 


\title{
Barkhausen pulses caused by domain merging in congruent lithium niobate
}

\author{
Cite as: Appl. Phys. Lett. 117, 022903 (2020); doi: 10.1063/5.0014220 \\ Submitted: 18 May 2020 - Accepted: 4 July 2020 . \\ Published Online: 15 July 2020

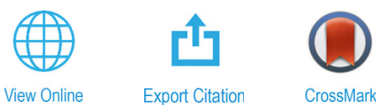

A. R. Akhmatkhanov, (D) I. A. Kipenko, A. A. Esin, (D) and V. Ya. Shur ${ }^{\text {a) }}$ (D)

\section{AFFILIATIONS}

School of Natural Sciences and Mathematics, Ural Federal University, Ekaterinburg 620000, Russia

a) Author to whom correspondence should be addressed: vladimir.shur@urfu.ru

\begin{abstract}
The analysis of the shapes of Barkhausen pulses (BPs) was used for the detailed characterization of the domain merging process in congruent lithium niobate $\mathrm{LiNbO}_{3}$ (CLN) crystals. The BPs in ferroelectrics manifest themselves as the sharp peaks in a switching current during polarization reversal by the application of a constant or slow-varying external electric field. Three mechanisms of the BPs were previously proposed: domain nucleation, interaction of the domain walls with the pinning centers, and domain merging. We have revealed the domination of the domain merging mechanism for the generation of the BPs in CLN and classified the scenarios of domain structure evolution after merging in terms of the appearance and transformation of short-lived fast and superfast domain walls. We have shown that the input of merging events reaches $80 \%$ of the whole switching process. Two revealed types of BPs corresponding to the merging events considerably differ by duration and shape, thus opening the way for solution of the inverse problem-extracting the quantitative information about the domain kinetics by the analysis of the BPs. This analysis allows extracting the velocities of the fast and superfast walls and provides the possibility to go beyond the temporal resolution of the in situ imaging system in studying the superfast domain wall motion. The proposed analysis is applicable for the faceted growth of polygonal domains in any ferroelectric. It is necessary to point out that the analysis of the BPs allowed characterizing the main part of the domain evolution process in CLN as the fast domain transformations after merging prevail during the polarization reversal.
\end{abstract}

Published under license by AIP Publishing. https://doi.org/10.1063/5.0014220

The Barkhausen effect discovered in ferromagnets in 1919 represents a sequence of the magnetization jumps resulting in the noise-like signal (Barkhausen noise) obtained during continuous magnetization or demagnetization and caused by the rapid changes of the domain sizes. ${ }^{1,2}$ The analysis of the Barkhausen noise has emerged as a valuable technique for studying the dynamics of domain formation and domain wall motion in ferromagnets. ${ }^{3,4}$

The similar effect representing the appearance of the Barkhausen pulses (BPs) in ferroelectrics was discovered in Rochelle salt ${ }^{5}$ and was later studied in potassium dihydrogen phosphate, ${ }^{6,7}$ barium titanate, ${ }^{8-10}$ lithium niobate, ${ }^{11}$ gadolinium molybdate, ${ }^{12,13}$ and lithium tantalate crystals. ${ }^{14}$ BPs manifest themselves as the sharp peaks in the switching current during the application of a constant or slow-varying external electric field. ${ }^{15,16}$

Later, it was shown that the Barkhausen noise represented an example of the self-organized criticality ${ }^{17}$ and could be analyzed in terms of avalanche statistics in both ferromagnets ${ }^{18-20}$ and ferroelectrics. ${ }^{21-24}$ This universal approach makes it possible to characterize, by critical exponents, the behavior of a broad range of natural phenomena, such as earthquakes, ${ }^{25}$ plastic deformations, ${ }^{26,27}$ vortex in superconductors, ${ }^{28}$ magnetic skyrmions, ${ }^{29}$ stars dimming, ${ }^{30}$ and even fluctuations in the financial markets. ${ }^{31}$ The detailed analysis of the discrete events allows revealing the universality class underlying the particular physical system. . $^{18,19,25}$

Initially, two mechanisms of BPs in ferroelectrics were proposed: (1) domain nucleation and fast forward growth ${ }^{9,15}$ and (2) interaction of the domain walls with the pinning centers in analogy with ferromagnets. ${ }^{4,15,16,32}$ Later, it was discovered by R. C. Miller that BPs in barium titanate crystals might occur when two growing domains came together. ${ }^{33}$ Through the direct observation of the domain structure and recording the switching current, the correlation of the BPs with the domain merging events ${ }^{34}$ and the shape transformations of the $90^{\circ}$ wedge domains were revealed. ${ }^{35}$

The analysis methods of the BPs in ferroelectrics can be divided into two groups: statistical methods and the analysis of the shape of the individual BP. The statistical methods based on the analysis of counting rate, duration, amplitude, and energy distribution functions of the BPs are more widespread due to their relative experimental 
simplicity (recording the switching current or acoustic emission ${ }^{13}$ is required only) and universality , $^{9,21-23,36-38}$ (see Ref. 21 for the comprehensive review). They allow characterizing a system in terms of the Hurst exponent, ${ }^{36}$ the Omori exponent, ${ }^{21}$ and the universality class. ${ }^{23}$

The analysis of shapes of the individual BPs is a far more complicated task from the experimental point of view as it requires additional in situ optical imaging of the domain structure kinetics with high temporal and spatial resolutions. ${ }^{22,39}$ The analysis of the BPs simultaneously recorded with the in situ domain imaging in congruent lithium niobate $\left(\mathrm{LiNbO}_{3}, \mathrm{CLN}\right)$ allowed revealing the direct correlation of the $\mathrm{BP}$ with the given domain merging event. ${ }^{39}$ However, the temporal resolution of the used imaging system $(15 \mathrm{~Hz})$ did not allow studying the fast domain kinetics. The recent studies of potassium titanyl phosphate $\left(\mathrm{KTiOPO}_{4}, \mathrm{KTP}\right)$ with a much higher temporal resolution $(4 \mathrm{kHz})$ revealed the formation and motion of the superfast domain walls after the domain merging and provided the wall velocity value by fitting of the corresponding $\mathrm{BP}{ }^{40}$

In this work, we have characterized, in detail, the domain merging process in CLN crystals by the analysis of the BP shapes. The periodically poled lithium niobate (PPLN) crystals with tailored precise stripe domain structures are widely used for various nonlinear optical applications. ${ }^{41,42}$ The uniaxial ferroelectric CLN crystals possess a simple domain structure, high crystal quality, and well-developed domain imaging techniques. ${ }^{43,44}$ The periodical poling is usually realized by the spatially nonuniform electric field produced by the stripe electrodes. However, broadening and merging of the growing domains outside the electrodes ${ }^{45}$ did not allow creating the submicrometer scale periods necessary for the realization of the new types of nonlinear optical interactions. ${ }^{46}$ The control of the domain merging by the analysis of the BPs opens the way for further development of the periodical poling. It is important that the proposed analysis is applicable for the faceted growth of the polygonal domains in any ferroelectric.

The studied samples represented the 0.5 -mm-thick plates of CLN single crystals (Gooch \& Housego, USA) cut perpendicular to the polar axis (Z-cut). The field application was realized using the $2-\mathrm{mm}$ in-diameter liquid (saturated aqueous solution of $\mathrm{LiCl}$ ) electrodes (see the supplementary material for details of the experimental setup). The rectangular field pulses with the amplitude $(E)$ from 20.7 to $22.4 \mathrm{kV} / \mathrm{mm}$ were used for the polarization reversal. The in situ imaging of the domain kinetics was realized using a high-speed CMOS camera Photron Mini UX100 (Photron, Japan) with a frame rate of $5000 \mathrm{~Hz}$.

The stack of the instantaneous domain pattern images obtained during the polarization reversal was digitally processed to enhance the domain wall contrast and overlapped into the single "kinetic map" image (see the supplementary material and Ref. 47).

The Cartesian and Miller-Bravais notations were used for the domain wall designation. We consider that the (hkil) wall propagates in the [hkil] direction; thus, we distinguish the (hkil) and (hkil) walls. We also denote the family of walls equivalent to the (hkil) wall within the crystal symmetry $\left(\mathrm{C}_{3 \mathrm{v}}\right)$ as $\{$ hkil $\}$.

The typical switching current obtained during the polarization reversal in CLN [Fig. 1(a), Multimedia view] contains more than 100 BPs (short peaks) separated by the low-current (below $40 \mu \mathrm{A}$ ) regions [Fig. 1(c)]. The switching charge of all BPs amounts to $85 \%$ of the total switching charge [Fig. 1(b)], indicating that the fast domain shape
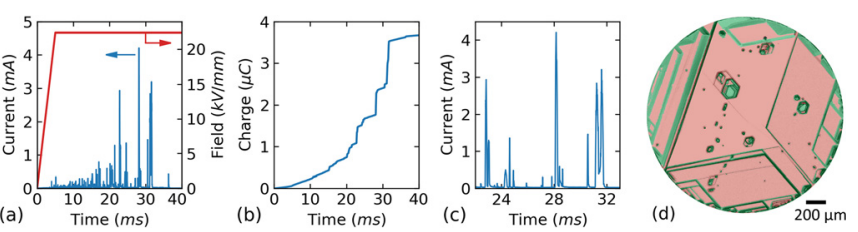

FIG. 1. (a) The shape of the switching current and the waveform of the applied external field. (b) The switching charge calculated by the integration of the switching current. (c) The magnified fragment of the switching current in (a). (d) The kinetic map of the switching process. Red-the regions switched by the motion of the fast and superfast walls appearing after the domain merging. Green-the regions switched by the motion of the slow walls. $E=22.4 \mathrm{kV} / \mathrm{mm}$. Multimedia view: https:// doi.org/10.1063/5.0014220.1

transformations after the merging prevail during the polarization reversal. It is necessary to point out that the switching charge of the highest $\mathrm{BP}$ is above $10 \%$ of the total switching charge [Fig. 1(b)].

Analysis of the corresponding kinetic map ${ }^{47}$ allows claiming that the most part of the area under the electrode is switched by the motion of the short-lived fast $\{1 \overline{4} 30\}$ and superfast $\{01 \overline{1} 0\}$ domain walls appearing after the domain merging [Fig. 1(d)]. It was shown that all the BPs in the switching current corresponded to these merging events representing the transformation of the domain shape from a concave polygon to a convex one. The fraction of the area switched by the motion of the fast and superfast domain walls [red regions in Fig. 1(d)] equals $80 \%$. This value is close to the estimation of the BPs input to the switched charge.

The merging events can be classified by the concave angles appearing after the merging. It is known that the polarization reversal in CLN for the used switching conditions (liquid electrodes and room temperature) leads to the formation and growth of the convex polygonal domains with the slow Y oriented $\{11 \overline{2} 0\}$ walls. ${ }^{48-50}$ Thus, the domain merging leads to the formation of the polygonal domains with $60^{\circ}$ and $120^{\circ}$ concave angles only [Fig. 2(a)]. For the $C_{3 v}$ symmetry of CLN, all the $60^{\circ}$ angles are equivalent, while the $120^{\circ}$ angles can be oriented in the $\mathrm{Y}+$ or $\mathrm{Y}$ - directions (the angle orientation is defined by the orientation of its bisecting line) [Fig. 2(a)]. Hereinafter, these $120^{\circ}$ angles will be designated as $120^{\circ} \mathrm{Y}+$ angle and $120^{\circ} \mathrm{Y}$ - angle, respectively.

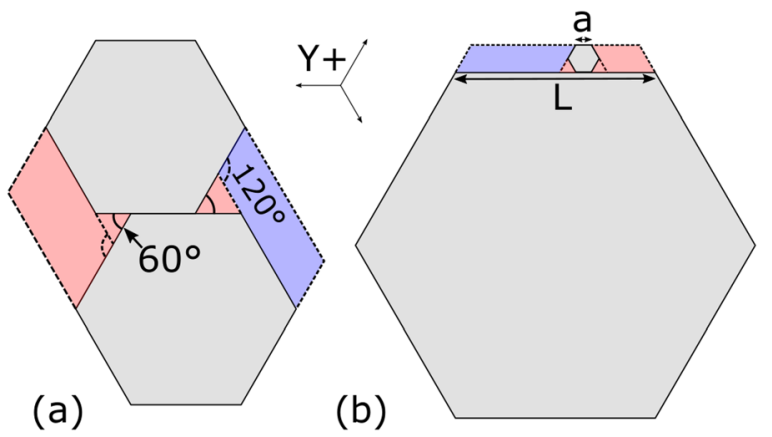

FIG. 2. Two scenarios of the hexagonal domain merging: (a) domains with comparable sizes (shape stability effect) and (b) a large domain with a small one (jerky wall motion effect). Red regions are switched by the motion of the superfast $\{01 \overline{1} 0\}$ domain wall, and blue regions are switched by the motion of the fast $\{1 \overline{4} 30\}$ domain wall. 
Our study of the formation and motion of the fast and superfast domain walls after merging is based on the analogy between the growth of ferroelectric domains and the crystal growth. ${ }^{51,52}$ The growth of an isolated domain being the convex growth is governed by the motion of the slowest domain walls. ${ }^{51}$ The merging of convex domains leads to the formation of the concave angles (less than $180^{\circ}$ in the direction of the advancing domain front), and the domain shape evolution is governed by the motion of the fastest walls. The type of the domain wall appearing in the given concave angle can be determined as the fastest wall in the corresponding sector of the kinetic Wulff plot, which has been recently reported for CLN. ${ }^{51}$ Thus, two types of the domain walls appear after the merging in CLN: (1) the superfast $\{01 \overline{1} 0\}$ wall for $60^{\circ}$ and $120^{\circ} \mathrm{Y}+$ angles and (2) the fast $\{1 \overline{4} 30\}$ wall for $120^{\circ}{ }_{Y-}$ angles (Fig. 2). The velocity of the superfast walls is about one order of magnitude higher than that of the fast ones. ${ }^{51}$

Two scenarios of the hexagonal domain merging can be distinguished. The first is the merging of the domains with comparable sizes, leading to the fast formation of the large convex hexagonal domain representing the so-called "shape stability effect" [Fig. 2(a)]..$^{53}$ The second is the merging of a large domain with a small one, leading to the effective acceleration of one slow wall resulting in the so-called "jerky wall motion effect" [Fig. 2(b)]. . $^{39}$

For the shape stability effect, two BPs are simultaneously generated after the merging: the short $\mathrm{BP}$ and the long $\mathrm{BP}$. The short $\mathrm{BP}$ is a result of the switching of the triangular and parallelogram regions by the single superfast domain wall [Figs. 3(b) and 3(c)]. The long BP is a result of the switching of the triangular region by the superfast domain wall and the parallelogram region by the fast one [Figs. 3(f) and 3(g)]. The experimentally observed short and long BPs and the corresponding in situ images of the domain kinetics are presented in Figs. $3(\mathrm{a})-3(\mathrm{~h})$. It is seen that the used imaging temporal resolution is insufficient to extract the positions of the superfast domain walls. During the analysis of the in situ images of the domain kinetics, it was taken into account that the jerky wall motion in CLN resulted in the formation of the wall traces [Figs. 3(a), 3(e), and 3(i)] corresponding to their rest positions. ${ }^{39,54,55}$

The simple analysis of the proper parameters of the BPs allows extracting the velocities of the fast and superfast domain walls (see the supplementary material),

$$
V_{s f}=\sqrt{\frac{j_{\max }^{s f}}{8 P_{s} t_{d e c}^{s f} \cos \left(\frac{\pi}{6}\right)}},
$$

where $j_{\text {max }}^{s f}$ is the maximum current and $t_{d e c}^{s f}$ is the decreased time of a fast BP [Figs. 3(c) and 3(d)], and $P_{s}$ is the spontaneous polarization

$$
V_{f}=\sqrt{\frac{j_{t d}^{f} \cos (\varphi) \cos \left(\varphi+\frac{\pi}{3}\right)}{4 P_{s} \cos \left(\frac{\pi}{6}\right) t_{t d}^{f}}},
$$

where $j_{t d}^{f}$ and $t_{t d}^{f}$ are the current value and time corresponding to the tilt decrease in slow BP [Figs. $3(\mathrm{~g})$ and $3(\mathrm{~h})$ ], respectively, and $\varphi=15^{\circ}$ is the tilt angle of the fast domain wall with respect to the $\{01 \overline{1} 0\}$ plane.

It is necessary to point out that the fitting of the short BPs corresponding to switching by the superfast wall requires deconvolution of the BP shape distorted by the recording system (see the supplementary material). ${ }^{15}$ The analysis of the presented BPs allowed obtaining the values of the superfast and fast domain wall velocities (Table I). The limited temporal resolution of the imaging system $(200 \mu \mathrm{s}$, corresponds to the frame rate $5000 \mathrm{~Hz}$ ) leads to the underestimation of the superfast domain wall velocity. It is clear that due to a higher temporal resolution $(3 \mu \mathrm{s}$, corresponds to the analog-to-digital converter recording rate $300 \mathrm{kHz}$ ), the values extracted by the $\mathrm{BP}$ analysis are more precise than those obtained by the in situ optical imaging (Table I).

The jerky wall motion effect appears as a result of merging when the side of the small hexagonal domain does not touch any vertex of the large one [Fig. 2(b)]. In terms of the kinetic approach to the domain wall motion, as a result of step generation and kink motion, ${ }^{56-58}$ the merging leads to the appearance of two macro-steps (echelons of elementary kinks): the first, with the superfast wall moving in the $\mathrm{Y}+$ direction, and the second, with the fast wall moving in the $\mathrm{Y}$ - direction [Figs. 3(i)-3(1)]. The completion of the merging process results in the fast shift of the slow domain wall by the distance $\Delta x=\sqrt{3} a$ (where $a$ is the side length of the small domain). The effective average slow wall velocity during this shift equals $\Delta x / \Delta t$, where $\Delta t$ is the duration of the gulping event. In the presented result [Figs. $3(i)-3(1)]$, the effective velocity of the slow wall (about $0.3 \mathrm{~m} / \mathrm{s}$ ) increased by three orders of magnitude as compared with the individual slow wall velocity for $E=22.4 \mathrm{kV} / \mathrm{mm}$ [about $0.5 \mathrm{~mm} / \mathrm{s}$ (Ref. 51)]. Moreover, the jerky motion event changes the domain shape by shortening the slow wall of the large hexagonal domain by $2 a$ [Fig. 2(b)].

We have shown that the domain merging is a dominating mechanism of the Barkhausen effect during polarization reversal in CLN single crystals. We have revealed and classified the main scenarios of the domain structure evolution in CLN after the domain merging in terms of the appearance and transformation of the short-lived fast and superfast domain walls. We have shown that the domain merging in ferroelectric with the $\mathrm{C}_{3 \mathrm{v}}$ symmetry leads to the formation of three types of the concave angles: $60^{\circ}, 120^{\circ} \mathrm{Y}+$, and $120^{\circ} \mathrm{Y}_{-}$. The study of the formation and motion of the fast and superfast domain walls was based on the analogy between the growth of crystals and ferroelectric domains. The orientation of the domain wall appearing in the given concave angle after the domain merging was determined as the fastest wall in the corresponding sector of the kinetic Wulff plot. Two scenarios of the hexagonal domain merging were distinguished. The first scenario is the merging of the domains with comparable sizes, leading to the fast formation of the large convex hexagonal domain representing the so-called shape stability effect. The second scenario is the merging of a large domain with a small one, leading to the effective acceleration of the slow wall resulting in the so-called jerky wall motion effect. The merging of the domains with comparable sizes leads to the appearance of two BPs: the short BP and the long BP. The short BP is a result of sequential switching in the $60^{\circ}$ and $120^{\circ} \mathrm{Y}+$ concave angles by the motion of the single superfast domain wall. The long BP is a result of switching in the $60^{\circ}$ concave angle by the motion of the superfast domain wall followed by switching in the $120^{\circ} \mathrm{Y}$ - concave angle by the fast wall. The two revealed types of the BPs corresponding to these merging events differ considerably by duration and shape, thus opening the way for solution of the inverse problem-extracting the quantitative information about the domain kinetics by the analysis of the BPs. The analysis of the shapes of short and long BPs has allowed extracting velocities of the fast and superfast walls with accuracy 


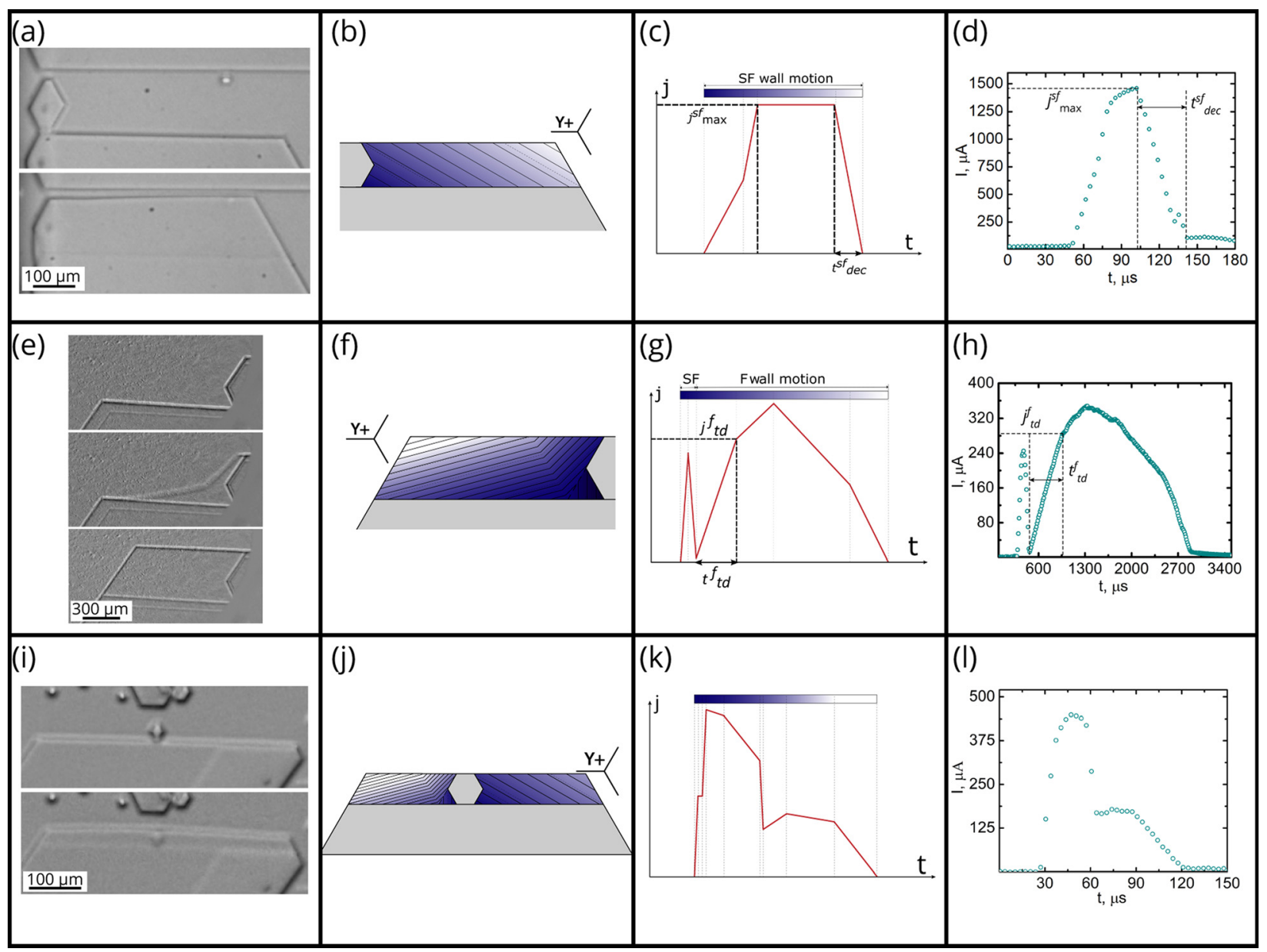

FIG. 3. The main types of the merging events in CLN. (a), (e), and (i) In situ images of the domain kinetics with time intervals (a), (i) $250 \mu \mathrm{s}$ and (e) $2 \mathrm{~ms}$. External field, $\mathrm{kV} / \mathrm{mm}$ : (a) 22.4, (e) 21.6, and (i) 22.4. (b), (f), and (j) The schemes of merging events. (c), (g), and (k) The schemes of the corresponding BPs. (d), (h), and (I) The shapes of the corresponding measured BPs.

outperforming the in situ imaging technique. The clearly revealed distinct behavior of the domain structure evolution during the short and long BPs points out on the possibility of the coexistence of different universality classes. The statistical analysis is required to justify this point. The control of the domain merging by the analysis of BPs is applicable for the faceted growth of polygonal domains in any ferroelectric and opens the ways for further development of the periodical poling. The merging of the neighboring stripe domains is the main problem of periodical poling, especially for short periods. Obviously, the merging is realized only by the motion of the fast and superfast walls. Thus, the analysis of BPs allows separating the inputs

TABLE I. The velocities of the superfast and fast domain walls obtained by the BP analysis and in situ imaging of the domain kinetics.

\begin{tabular}{lcc}
\hline \hline & BP analysis & In situ imaging \\
\hline Superfast, $\mathrm{m} / \mathrm{s}$ & $3.16 \pm 0.19$ & $1.6 \pm 0.8$ \\
Fast, $\mathrm{m} / \mathrm{s}$ & $0.243 \pm 0.009$ & $0.13 \pm 0.03$ \\
\hline
\end{tabular}

corresponding to the fast and superfast walls by detecting the merging events. Eventually, this analysis will allow optimizing the poling parameters and improve the quality of obtained periodical domain structures for short-pitch domain patterns.

See the supplementary material for the experimental details and calculation of the fast and superfast domain wall velocities.

The equipment of the Ural Center for Shared Use "Modern nanotechnology" UrFU was used. The research was made possible by the Russian Science Foundation (Project No. 19-72-00083).

\section{DATA AVAILABILITY}

The data that support the findings of this study are available from the corresponding author upon reasonable request.

\section{REFERENCES}

${ }^{1}$ H. Barkhausen, Phys. Z. 20, 401 (1919).

${ }^{2}$ H. J. Williams and W. Shockley, Phys. Rev. 75, 178 (1949). 
${ }^{3}$ S. Santa-aho, A. Laitinen, A. Sorsa, and M. Vippola, J. Nondestr. Eval. 38, 94 (2019).

${ }^{4}$ V. M. Rudyak, Sov. Phys. Usp. 13, 461 (1971)

${ }^{5}$ H. Mueller, Phys. Rev. 47, 175 (1935).

${ }^{6}$ W. Bantle and A. V. Arx, Helv. Phys. Acta 17, 298 (1944).

${ }^{7}$ B. Zwicker and P. Scherrer, Helv. Phys. Acta 17, 346 (1944).

${ }^{8}$ A. C. Kibblewhite, Proc. IEE 102, 59 (1955).

${ }^{9}$ A. G. Chynoweth, Phys. Rev. 110, 1316 (1958).

${ }^{10}$ R. R. Newton, A. J. Ahearn, and K. G. McKay, Phys. Rev. 75, 103 (1949).

${ }^{11}$ V. I. Kovalevich, L. A. Shuvalov, and T. R. Volk, Phys. Status Solidi A 45, 249 (1978).

${ }^{12}$ V. Ya. Shur, E. V. Nikolaeva, E. L. Rumyantsev, E. I. Shishkin, A. L. Subbotin, and V. L. Kozhevnikov, Ferroelectrics 222, 323 (1999).

${ }^{13}$ D. C. Lupascu, V. Ya. Shur, and A. G. Shur, Appl. Phys. Lett. 80, 2359 (2002).

${ }^{14}$ V. Ya. Shur, A. R. Akhmatkhanov, I. S. Baturin, and E. V. Shishkina, J. Appl. Phys. 111, 014101 (2012).

${ }^{15}$ A. G. Chynoweth, J. Appl. Phys. 30, 280 (1959).

${ }^{16} \mathrm{M}$. Lines and A. Glass, Principles and Applications of Ferroelectrics and Related Materials (Clarendon Press, Oxford, 1979).

${ }^{17}$ P. Bak, C. Tang, and K. Wiesenfeld, Phys. Rev. A 38, 364 (1988).

${ }^{18}$ F. Bohn, G. Durin, M. A. Correa, N. R. Machado, R. D. Della Pace, C. Chesman, and R. L. Sommer, Sci. Rep. 8, 11294 (2018).

${ }^{19}$ D. M. Silevitch, J. Xu, C. Tang, K. A. Dahmen, and T. F. Rosenbaum, Phys. Rev. B 100, 134405 (2019).

${ }^{20}$ B. Tadić, S. Mijatović, S. Janićević, D. Spasojević, and G. J. Rodgers, Sci. Rep. 9, 6340 (2019).

${ }^{21}$ C. Flannigan, C. D. Tan, and J. F. Scott, J. Phys. 32, 055403 (2020).

${ }^{22}$ B. Casals, G. F. Nataf, D. Pesquera, and E. K. H. Salje, APL Mater. 8, 011105 (2020).

${ }^{23}$ C. D. Tan, C. Flannigan, J. Gardner, F. D. Morrison, E. K. H. Salje, and J. F. Scott, Phys. Rev. Mater. 3, 034402 (2019).

${ }^{24}$ E. K. H. Salje, D. Xue, X. Ding, K. A. Dahmen, and J. F. Scott, Phys. Rev. Mater. 3, 014415 (2019).

${ }^{25}$ J. P. Sethna, K. A. Dahmen, and C. R. Myers, Nature 410, 242 (2001).

${ }^{26}$ F. F. Csikor, C. Motz, D. Weygand, M. Zaiser, and S. Zapperi, Science 318, 251 (2007).

${ }^{27}$ D. M. Dimiduk, C. Woodward, R. LeSar, and M. D. Uchic, Science 312, 1188 (2006).

${ }^{28}$ S. Field, J. Witt, F. Nori, and X. Ling, Phys. Rev. Lett. 74, 1206 (1995).

${ }^{29}$ S. A. Díaz, C. Reichhardt, D. P. Arovas, A. Saxena, and C. J. O. Reichhardt, Phys. Rev. Lett. 120, 117203 (2018).

${ }^{30}$ M. A. Sheikh, R. L. Weaver, and K. A. Dahmen, Phys. Rev. Lett. 117, 261101 (2016).
${ }^{31}$ T. Lux and M. Marchesi, Nature 397, 498 (1999).

${ }^{32}$ V. Ya. Shur, E. L. Rumyantsev, V. P. Kuminov, A. L. Subbotin, and V. L. Kozhevnikov, Phys. Solid State 41, 269 (1999).

${ }^{33}$ R. C. Miller, Phys. Rev. 111, 736 (1958).

${ }^{34}$ R. C. Miller, J. Phys. Chem. Solids 17, 93 (1960).

${ }^{35}$ B. Brezina, J. Fousek, and A. Glanc, Czech. J. Phys. B 11, 595 (1961)

${ }^{36}$ V. Ya. Shur, E. L. Rumyantsev, D. V. Pelegov, V. L. Kozhevnikov, E. V. Nikolaeva, E. L. Shishkin, A. P. Chernykh, and R. K. Ivanov, Ferroelectrics 267, 347 (2002).

${ }^{37}$ D. C. Lupascu, T. Granzow, and T. Woike, Europhys. Lett. 68, 733 (2004).

${ }^{38}$ E. Dul'kin, J. Zhai, and M. Roth, Phys. Status Solidi B 252, 2079 (2015).

${ }^{39}$ I. S. Baturin, M. V. Konev, A. R. Akhmatkhanov, A. I. Lobov, and V. Ya. Shur, Ferroelectrics 374, 136 (2008).

${ }^{40}$ A. R. Akhmatkhanov, A. A. Esin, E. M. Vaskina, M. A. Alam, and V. Ya. Shur, Ferroelectrics 525, 11 (2018).

${ }^{41}$ R. L. Byer, J. Nonlinear Opt. Phys. Mater. 06, 549 (1997).

${ }^{42}$ D. S. Hum and M. M. Fejer, C. R. Phys. 8, 180 (2007).

${ }^{43}$ E. Soergel, Appl. Phys. B 81, 729 (2005).

${ }^{44}$ V. Ya. Shur and P. S. Zelenovskiy, J. Appl. Phys. 116, 066802 (2014).

${ }^{45}$ G. Rosenman, Kh. Garb, A. Skliar, M. Oron, D. Eger, and M. Katz, Appl. Phys. Lett. 73, 865 (1998).

${ }^{46}$ C. E. Minor and R. S. Cudney, Appl. Phys. B 123, 38 (2017).

${ }^{47}$ V. Ya. Shur, A. A. Esin, M. A. Alam, and A. R. Akhmatkhanov, Appl. Phys. Lett. 111, 152907 (2017).

${ }^{48}$ V. Gopalan, Q. X. Jia, and T. E. Mitchell, Appl. Phys. Lett. 75, 2482 (1999).

${ }^{49}$ A. A. Esin, A. R. Akhmatkhanov, and V. Ya. Shur, Appl. Phys. Lett. 114, 092901 (2019).

${ }^{50}$ B. Kirbus, C. Godau, L. Wehmeier, H. Beccard, E. Beyreuther, A. Haußmann, and L. M. Eng, ACS Appl. Nano Mater. 2, 5787 (2019).

${ }^{51}$ A. A. Esin, A. R. Akhmatkhanov, and V. Ya. Shur, Appl. Phys. Lett. 114, 192902 (2019).

${ }^{52}$ A. A. Esin, A. R. Akhmatkhanov, and V. Ya. Shur, J. Cryst. Growth 526, 125236 (2019).

${ }^{53}$ V. Ya. Shur, A. R. Akhmatkhanov, D. S. Chezganov, A. I. Lobov, I. S. Baturin, and M. M. Smirnov, Appl. Phys. Lett. 103, 242903 (2013).

${ }^{54}$ V. Ya. Shur, A. R. Akhmatkhanov, I. S. Baturin, M. S. Nebogatikov, and M. A. Dolbilov, Phys. Solid State 52, 2147 (2010).

${ }^{55} \mathrm{M}$. Müller, E. Soergel, and K. Buse, Opt. Lett. 28, 2515 (2003).

${ }^{56}$ V. Ya. Shur, J. Mater. Sci. 41, 199 (2006).

${ }^{57}$ J. Gonnissen, D. Batuk, G. F. Nataf, L. Jones, A. M. Abakumov, S. Van Aert, D. Schryvers, and E. K. H. Salje, Adv. Funct. Mater. 26, 7599 (2016).

${ }^{58}$ L. Zhang, S. Li, X. Ding, J. Sun, and E. K. H. Salje, Appl. Phys. Lett. 116, 102902 (2020). 